\title{
PRECISION ONCOLOGY IN SURGERY: PATIENT SELECTION FOR OPERABLE RECURRENT HEPATIC OVARIAN CANCER
}

\section{Conte ${ }^{1}$, V. Gallotta ${ }^{1}$, M. D'Indinosante ${ }^{2}$, E. Capoluongo ${ }^{3-4}$, A. Minucci ${ }^{4}$, A. De Rose ${ }^{5}, \mathrm{~F}$. Ardito ${ }^{5}$, F.}

Giuliante ${ }^{5}$, A. Di Giorgio 6 ${ }^{6}$, G.F. Zannoni ${ }^{7}$, C. Margreiter ${ }^{8}$, A. Fagotti ${ }^{1-2}$, G. Scambia ${ }^{1-2}$, G. Ferrandina ${ }^{1-9}$

1) Fondazione Policlinico Universitario A. Gemelli, IRCCS, UOC Ginecologia Oncologica, Dipartimento per la salute della Donna e del Bambino e della Salute Pubblica, Roma, Italia;

2) Università Cattolica del Sacro Cuore, Istituto di Ginecologia e Ostetricia, Roma, Italia,

3) Laboratory of Clinical Molecular and Personalized Diagnostics, Catholic University of the Sacred Heart, Roma, Italy;

4) Laboratory of Clinical Molecular and Personalized Diagnostics, Fondazione Policlinico Universitario A. Gemelli IRCCS, Rome, Italy;

5) Department of Surgical Sciences, Hepatobiliary Unit, Fondazione Policlinico Universitario A. Gemelli IRCCS, Catholic University of the Sacred Heart, Rome, Italy;

6) Division of General Surgery, Fondazione Policlinico Universitario A. Gemelli IRCCS, Rome, Italyglnstitute of Histopathology, Fondazione Policlinico Universitario A Gemelli

IRCCS, Catholic University of the Sacred Heart, Roma, Italy;

7) Institute of Histopathology, Fondazione Policlinico Universitario A. Gemelli IRCCS, Catholic University of the Sacred Heart, Roma, Italy;

8) Department of Visceral, Transplant, and Thoracic Surgery, Medical University of Innsbruck, Innsbruck, Austria;

9) Gynecologic Oncology, Centro di Ricerca e Formazione ad Alta Tecnologia nelle Scienze Biomediche, Universita Cattolica del Sacro Cuore, Campobasso, Italy.

\section{OBJECTIVE}

- To describe the oncological outcomes after hepatic resection (HR) in recurrent ovarian carcinoma (ROC) evaluating clinic-pathological variables and mutational status of BRCA

- Although HR is considered a challenging situation in ROC patients, assessment of BRCA1/2 mutational status seems to have a relevant clinical value to guide surgical therapy.

\section{METHODS}

Patients who underwent HR for ROC at the Catholic University of Rome, between June 2012 and October 2017 were included.

Exclusion criteria were represented by extra-abdominal disease and presence of diffuse peritoneal

carcinomatosis requiring more than 2 bowel resections.

\section{RESULTS}

- Thirty-four patients undewent HR within secondary cytoreductive surgery (SCS).

- Six patients (17.6\%) presented with hepatic relapse only, while the remaining 28 patients $(82.4 \%)$ had concomitant extra-hepatic disease.

- In the whole series, the 3-yr PHR-PFS was $49.1 \%$ and the $3-y r$ post-HR overall survival was $72.9 \%$.

- Uni-variate analysis of variables conditioning PHR-PFS showed that only BRCA mutational status played a statistically significant favorable role: the 3-yr PHR-PFS rate was $81.0 \%$ in BRCA mutated patient compared to $15.2 \%$ in wild type ones ( $p$ value: 0.001 )
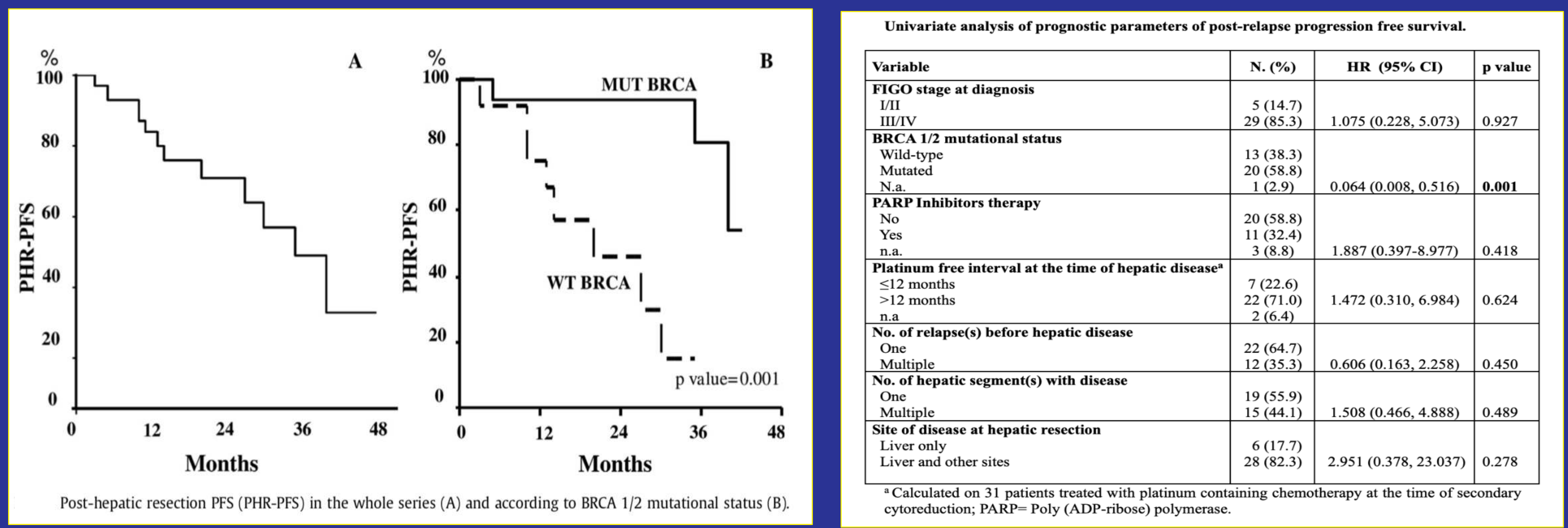

\section{CONCLUSION}

Our clinical analyses suggest that in ROC patients with liver disease the assessment of germline and somatic BRCA mutational status can help to select patients eligible for SCS. 